\title{
Avaliação do conhecimento dos estudantes de Odontologia da UFSM sobre DTM e bruxismo
}

\author{
Gabriela Rahmeier*; Gabriella Lenz Irineu*; Lucas Machado Maracci**; Rafaela Stocker Salbego*; \\ Luiza Pereira do Nascimento***; Mariana Marquezan****; Gabriela Salatino Liedke ${ }^{* * * * *}$; Tatiana \\ Bernardon Silva ${ }^{* * * * *}$
}

* Graduada em Odontologia, Universidade Federal de Santa Maria

** Mestrando em Ciências Odontológicas, Universidade Federal de Santa Maria

*** Doutoranda em Ciências Odontológicas, Universidade Federal de Santa Maria

**** Professora adjunta, Departamento de Estomatologia, Universidade Federal de Santa Maria

****** Professora Titular, Departamento de Odontologia Restauradora, Universidade Federal de Santa Maria

Recebido: 10/12/2020. Aprovado: 18/05/2021.

\begin{abstract}
RESUMO
Casos de bruxismo e disfunção temporomandibular (DTM) têm se tornado cada vez mais frequentes na prática clínica. Reconhecer seus mecanismos é necessário para o sucesso do controle e tratamento. O objetivo deste estudo piloto foi investigar o conhecimento e a percepção sobre DTM e bruxismo dos estudantes de Odontologia da Universidade Federal de Santa Maria (UFSM). Para isso um questionário com 15 questões foi aplicado a 20 estudantes dessa instituição, sendo 10 do $7^{\circ}$ e 10 do $10^{\circ}$ semestre. Todos os estudantes avaliados relataram ouvir sobre DTM durante a graduação, mas $70 \%$ consideraram que possuíam pouca ou nenhuma base de conhecimento sobre o assunto. Noventa por cento dos estudantes relatou não conhecer a ferramenta utilizada em pesquisas para diagnóstico das DTM. Não houve diferença estatística entre os grupos avaliados e a média geral de acertos foi de $54 \%$. Os dados evidenciam o pouco conhecimento sobre os assuntos abordados e a fragilidade do ensino destas alterações, especialmente pela não evolução do conhecimento sobre DTM e bruxismo ao longo do curso avaliado, pois o grupo de estudantes do $10^{\circ}$ semestre apresentou resultados inferiores quando comparados ao do $7^{\circ}$ semestre, que passaram recentemente pela Clínica de Oclusão, na qual os temas são abordados.
\end{abstract}

Descritores: Síndrome da Disfunção da Articulação Temporomandibular. Bruxismo. Inquéritos e Questionários. Estudantes de Odontologia. Avaliação Educacional. 


\section{INTRODUÇÃO}

Define-se bruxismo como a ação repetitiva dos músculos da mastigação, levando ao apertar ou ranger de dentes e/ou travamento ou projeção da mandíbula ${ }^{1,2}$. Pode-se classificá-lo como bruxismo em vigília (BV) quando ocorre com o paciente acordado, ou como bruxismo do sono (BS), quando o paciente está dormindo ${ }^{1,2}$. Além disso, essas condições são consideradas fatores de risco para desenvolvimento de disfunções temporomandibulares (DTM). As DTM abrangem uma série de alterações funcionais nos músculos da mastigação, articulação temporomandibular (ATM) e estruturas orofaciais associadas ${ }^{3}$. Sua etiologia é multifatorial e complexa, incluindo traumas, fatores genéticos, gatilhos cognitivos, estresse emocional e fatores biológicos, como hiperatividade muscular ${ }^{4,5}$. Essas disfunções predominam no sexo feminino e tipicamente apresentam um curso recorrente e crônico, sendo a diminuição da amplitude de abertura bucal, a presença de trigger points $\mathrm{e}$ as dores os principais sinais e sintomas relatados pelos pacientes ou encontrados pelo cirurgião-dentista ${ }^{5,6}$. Frequentemente, bruxismo e DTM são confundidos ou englobados, de maneira errônea, em uma mesma condição clínica, visto que apresentam etiologias diferentes e por isso precisam de protocolos clínicos distintos.

DTM e dor orofacial são importantes componentes da nova filosofia da Odontologia com foco não apenas nos dentes, mas sim no paciente como um todo. Porém, essas condições foram consideradas uma especialidade há poucos anos. Em 1993, a International Association for the Study of Pain (IASP) propôs pela primeira vez o "estudo da dor" nos currículos de graduação em Odontologia ${ }^{6,7}$. No entanto, até hoje o modelo adotado pelas escolas de Odontologia possui pouca carga horária para adaptar os novos conteúdos do estudo da dor ${ }^{6}$.
Ademais, o estudo acerca das DTM quase nunca é ministrado por professores especialistas no assunto, e sim, em sua maioria, por professores de prótese dentária ${ }^{8}$. Na instituição avaliada, o estudo das DTM e bruxismo são ministrados na Clínica de Oclusão.

Aproximadamente 40 a 50\% da população apresenta algum sinal ou sintoma de DTM, contudo apenas $5 \%$ dos indivíduos procuram por tratamento9 ${ }^{9}$. Destes, muitos são tratados como portadores de uma dor de origem odontogênica, pela falta de conhecimento da complexidade dos mecanismos da dor pelo cirurgião-dentista, resultando em iatrogenias e falhas no tratamento, como piora na sintomatologia ou relato do surgimento de uma nova dor. Apesar de se tratar de um assunto de extrema relevância, a falta de padronização dos currículos acadêmicos dificulta a aquisição e a implementação de conhecimentos sobre dor orofacial ${ }^{10}$, sem haver enfoque no ensinamento da dor ${ }^{11}$, o que resulta na baixa confiança dos acadêmicos para tratar pacientes com DTM ${ }^{12}$.

Assim, o presente estudo piloto teve como objetivo comparar estudantes de diferentes semestres em uma faculdade de Odontologia a fim de avaliar seus conhecimentos e percepções sobre o conteúdo de DTM e bruxismo experienciado durante a graduação.

\section{MÉTODOS}

Este estudo piloto observacional de natureza transversal foi aprovado pelo Comitê de Ética da Universidade Federal de Santa Maria (CEP-UFSM) no ano de 2019 (CAAE 24282719.1.1001.5346, protocolo 052935).

A instituição escolhida inclui em seu currículo o estudo sobre DTM e bruxismo na disciplina de Clínica de Oclusão, lotada no Departamento de Odontologia Restauradora e ofertada no $6^{\circ}$ semestre. Possui carga horária total de $60 \mathrm{~h}$, sendo $45 \mathrm{~h}$ destinadas a atividades 
práticas como moldagem, uso do articulador semiajustável e enceramento da placa de Michigan, e $15 \mathrm{~h}$ referentes a aulas teóricas expositivo-dialogadas e estudo de casos clínicos. Seu conteúdo programático é dividido em 5 unidades: 1) Introdução ao Estudo da Oclusão Dentária; 2) Anatomia e Fisiologia do Sistema Estomatognático; 3) Princípios de Oclusão; 4) Aplicação dos Princípios de Oclusão a Outras Especialidades; e 5) Desordens Temporomandibulares (conceito, etiologia, diagnóstico, classificação, tratamento e bruxismo).

Com o objetivo de avaliar o conhecimento e a percepção dos estudantes sobre as DTM e bruxismo, foram selecionados estudantes do $7^{\circ}$ semestre, pelo fato de recentemente terem realizado a disciplina de Clínica de Oclusão e estudantes do $10^{\circ}$ semestre, por estarem cursando o último semestre da graduação. Apenas 20 deles (32,78\% de um total de 61 alunos), sendo 10 de cada semestre selecionado, responderam a um questionário, já que a coleta de dados iniciou se deu pouco antes do início da pandemia causada pela COVID-19. Cada participante recebeu e assinou o Termo de Consentimento Livre e Esclarecido, ficando com uma cópia para si.

O questionário foi composto por 15 questões, com perguntas fechadas (objetivas) e abertas (discursivas). Os três primeiros questionamentos foram de cunho pessoal, onde o estudante deveria marcar "sim" ou "não" para informar se durante a sua graduação ouviu falar em DTM e se conhecia tal termo. Logo após, o estudante deveria classificar o nível de conhecimento que a instituição oferece sobre DTM durante a graduação, sendo as opções "pouca ou nenhuma base" e "profundo". As questões seguintes foram distribuídas em seções, sendo cinco delas para avaliar o conhecimento sobre etiologia e sintomatologia das DTM e bruxismo e outras sete sobre a atitude dos estudantes para possíveis tratamentos frente a situações específicas. As perguntas foram desenvolvidas pelos autores, com base em livros e artigos considerados referências no estudo de DTM e bruxismo. Os participantes não tiveram acesso a materiais de consulta, impresso ou eletrônico, para a resolução do questionário.

Após a realização do questionário, os dados foram coletados, calculou-se a porcentagem de acerto dos grupos em cada questão e os resultados foram obtidos a partir do teste exato de Fisher. Este foi o método de escolha, pois se aplica a todos os tamanhos amostrais de pesquisa. Foi considerada uma significância estatística de 5\%.

\section{RESULTADOS}

O questionário foi respondido por 20 estudantes $\left(10\right.$ do $10^{\circ}$ semestre e 10 do $7^{\circ}$ semestre), separados em dois grupos. Na tabela 1, estão listados os resultados obtidos nas três primeiras questões, pessoais e autoavaliativas. Os resultados da seção seguinte, cujas cinco questões abordaram o conhecimento sobre DTM e bruxismo, são mostrados na tabela 2. O resultado de outras sete questões, relacionadas à seção sobre atitudes, estão listados na tabela 3.

Noventa por cento dos estudantes, em ambos os grupos, relataram não conhecer a ferramenta utilizada em pesquisa para diagnóstico das disfunções temporomandibulares citada na questão 8 - o Reserch Diagnostic Criteria of Temporomandibular Disorders (RDC/TMD). Aqueles que relataram ter conhecimento seguiram para a parte descritiva da questão, detalhando aquilo que conheciam a respeito. Ao final da pesquisa, quando somada a média de acertos em cada grupo, o resultado ficou em $60 \%$ de acertos para o $7^{\circ}$ semestre e de $49 \%$ para o $10^{\circ}$ semestre. No geral, a média de acertos foi de $54 \%$. 
Tabela 1. Respostas dos estudantes às questões autoavaliativas

\begin{tabular}{lcc}
\hline Questões & $\begin{array}{c}\mathbf{7}^{\mathbf{0}} \text { semestre } \\
\mathbf{n}(\boldsymbol{\%})\end{array}$ & $\begin{array}{c}\mathbf{1 0}^{\mathbf{0}} \text { semestre } \\
\mathbf{n}(\boldsymbol{\%})\end{array}$ \\
\hline $\begin{array}{l}\text { 1. Você já ouviu falar durante a sua graduação em disfunções } \\
\text { temporomandibulares? }\end{array}$ & $10(100) \operatorname{sim}$ & $10(100)$ sim \\
$\begin{array}{l}\text { 2. Você sabe o que é disfunção temporomandibular? } \\
\text { 3. De acordo com sua opinião, que nível de conhecimento de avaliação é } \\
\text { fornecido durante a graduação em relação às disfunções }\end{array}$ & $\begin{array}{c}6(60) \text { pouca ou } \\
\text { temporomandibulares? }\end{array}$ & $\begin{array}{c}\text { nenhuma base }(80) \text { pouca ou } \\
\text { nenhuma base }\end{array}$ \\
\hline
\end{tabular}

Tabela 2. Resultados obtidos na avaliação da seção conhecimento

\begin{tabular}{|c|c|c|c|}
\hline Questões & $\begin{array}{c}7^{\circ} \text { semestre } \\
\text { Acertos } n(\%)\end{array}$ & $\begin{array}{c}10^{\circ} \text { semestre } \\
\text { Acertos } n(\%)\end{array}$ & $\begin{array}{l}\text { Valor } \\
\text { de } \mathbf{p}^{*}\end{array}$ \\
\hline $\begin{array}{l}\text { 4. Bruxismo é o tipo de disfunção temporomandibular mais } \\
\text { prevalente na população atualmente? } \\
\text { Resposta correta: DISCORDO }\end{array}$ & $4(40)$ & $4(40)$ & 0,429 \\
\hline \multicolumn{4}{|l|}{$\begin{array}{l}\text { 5. Quais dos seguintes itens contribuem para a etiologia das } \\
\text { disfunções temporomandibulares? }\end{array}$} \\
\hline \multicolumn{4}{|l|}{ Resposta correta: TODOS } \\
\hline Sofrimento emocional & $9(90)$ & $8(80)$ & 0,598 \\
\hline Trauma na região maxilofacial & $7(70)$ & $5(50)$ & 0,490 \\
\hline Bruxismo do sono & $8(80)$ & $8(80)$ & 0,236 \\
\hline Bruxismo da vigília & $8(80)$ & $8(80)$ & 0,236 \\
\hline Postura corporal anormal & $7(70)$ & $5(50)$ & 0,490 \\
\hline Respiração bucal & $7(70)$ & $5(50))$ & 0,490 \\
\hline Maloclusão & $8(80)$ & $8(80)$ & 0,429 \\
\hline Genética & $4(40)$ & $1(10)$ & 0,389 \\
\hline Traumatismo por hiperextensão & $4(40)$ & $5(50)$ & 1,000 \\
\hline Associado a outros distúrbios musculoesqueléticos & $7(70)$ & $8(80)$ & $\mathbf{0 , 0 1 6}$ \\
\hline \multicolumn{4}{|l|}{$\begin{array}{l}\text { 6. Quais os indivíduos mais afetados pelas disfunções } \\
\text { temporomandibulares? }\end{array}$} \\
\hline \multicolumn{4}{|l|}{ Resposta correta: INDIVÍDUOS JOVENS E DE MEIA IDADE } \\
\hline Indivíduos jovens & $4(40)$ & $4(40)$ & 0,598 \\
\hline Indivíduos de meia idade & $8(80)$ & $5(50)$ & 1,000 \\
\hline Indivíduos idosos & $10(100)$ & $8(80)$ & - \\
\hline $\begin{array}{l}\text { 7. Um indivíduo com disfunção temporomandibular pode } \\
\text { apresentar os sintomas de: } \\
\text { Resposta correta: TODOS }\end{array}$ & & & - \\
\hline Dor na região pré-auricular & $10(100)$ & $8(80)$ & - \\
\hline Dificuldade em abrir a boca & $9(90)$ & $9(90)$ & 0,725 \\
\hline Sons articulares & $10(100)$ & $8(80)$ & - \\
\hline Dor muscular & $9(90)$ & $10(100)$ & - \\
\hline Dor referida na região cervical & $9(90)$ & $5(50)$ & 0,292 \\
\hline Via alterada da abertura da boca & $8(80)$ & $6(60)$ & 0,747 \\
\hline
\end{tabular}

*Teste Exato de Fisher 
Tabela 3. Resultados obtidos na avaliação da seção atitude

\begin{tabular}{|c|c|c|c|}
\hline Questões & $\begin{array}{c}7^{0} \text { semestre } \\
\text { Acertos } n(\%)\end{array}$ & $\begin{array}{c}10^{\circ} \text { semestre } \\
\text { Acertos } n(\%)\end{array}$ & $\begin{array}{l}\text { Valor } \\
\text { de } \text { p }^{*}\end{array}$ \\
\hline $\begin{array}{l}\text { 9. Identificação e remoção de interferências oclusais são eficazes no } \\
\text { tratamento das disfunções temporomandibulares? } \\
\text { Resposta correta: DISCORDO }\end{array}$ & $3(30)$ & $2(20)$ & 0,301 \\
\hline $\begin{array}{l}\text { 10. O tratamento ortodôntico deve ser opção de escolha no tratamento } \\
\text { das disfunções temporomandibulares? } \\
\text { Resposta correta: DISCORDO }\end{array}$ & $7(70)$ & $8(80)$ & 0,490 \\
\hline $\begin{array}{l}\text { 11. O tratamento ortodôntico pode ser iniciado em pacientes com } \\
\text { distúrbios da articulação temporomandibular? } \\
\text { Resposta correta: DISCORDO }\end{array}$ & $4(40)$ & $6(60)$ & 0,598 \\
\hline $\begin{array}{l}\text { 12. Todos os indivíduos com ruídos articulares não requerem } \\
\text { tratamento? } \\
\text { Resposta correta: DISCORDO }\end{array}$ & $10(100)$ & $9(90)$ & - \\
\hline $\begin{array}{l}\text { 13. Todos os indivíduos com DTM não precisam passar por avaliação } \\
\text { radiográfica antes da formulação do tratamento? } \\
\text { Resposta correta: } D I S C O R D O\end{array}$ & $10(100)$ & $9(90)$ & - \\
\hline $\begin{array}{l}\text { 14. A toxina botulínica é um dos principais tratamentos para as } \\
\text { disfunções temporomandibulares musculares? } \\
\text { Resposta correta: DISCORDO }\end{array}$ & $9(90)$ & $5(50)$ & 0,292 \\
\hline $\begin{array}{l}\text { 15. As cirurgias de recaptura de disco são muito eficientes em casos de } \\
\text { pacientes com deslocamento de disco com redução? } \\
\text { Resposta correta: DISCORDO }\end{array}$ & $9(70)$ & $4(40)$ & 0,260 \\
\hline
\end{tabular}

Noventa por cento dos estudantes, em ambos os grupos, relataram não conhecer a ferramenta utilizada em pesquisa para diagnóstico das disfunções temporomandibulares citada na questão 8 - o Reserch Diagnostic Criteria of Temporomandibular Disorders (RDC/TMD). Aqueles que relataram ter conhecimento seguiram para a parte descritiva da questão, detalhando aquilo que conheciam a respeito. Ao final da pesquisa, quando somada a média de acertos em cada grupo, o resultado ficou em $60 \%$ de acertos para o $7^{\circ}$ semestre e de $49 \%$ para o $10^{\circ}$ semestre. No geral, a média de acertos foi de $54 \%$.

\section{DISCUSSÃO}

$\mathrm{Na}$ perspectiva dos autores, os estudantes apresentaram pouco conhecimento sobre DTM e bruxismo devido à insuficiente carga horária ofertada para estudo desses assuntos. Não houve ampliação do conhecimento do $7^{\circ}$ semestre para o $10^{\circ}$. O curso avaliado apresenta estrutura curricular ampla e recentemente reformulada em $2017^{13}$ e não apresenta disciplinas específicas que sejam voltadas para o estudo das disfunções temporomandibulares e do bruxismo.

Em 2011, uma pesquisa realizada em 53 cursos de Odontologia do Brasil mostrou que menos de 5\% da carga horária total, em média, era dedicada ao estudo da dor ${ }^{8}$. Os conteúdos estão, de forma geral, distribuídos nos componentes curriculares básicos, como Fisiologia e Anatomia, e clínicos, como Farmacologia, Patologia e Endodontia. O mesmo acontece com o estudo de DTM: os responsáveis pelo seu ensino não são profissionais especialistas no assunto, e sim professores de prótese dentária na grande 
maioria ${ }^{8}$. Ainda, estudos indicam que a prática clínica logo após o estudo teórico sobre DTM aumenta o conhecimento dos estudantes ${ }^{7}$. Identificar fatores de risco, realizar diagnóstico, planejar tratamentos e agir em cooperação com uma equipe multidisciplinar são competências alcançadas através do treinamento clínico ${ }^{10}$.

Em ambos os grupos avaliados, $60 \%$ dos estudantes concordaram equivocadamente com a afirmação de que "o bruxismo é o principal tipo de DTM". O bruxismo pode levar os músculos mastigatórios a um estado de hiperfunção, causando uma sobrecarga nas articulações temporomandibulares e, consequentemente, ocasionando dor muscular e articular ${ }^{16,17}$. O bruxismo em vigília é um fator de risco significativo para a ocorrência de DTM, por ser seis vezes mais frequente que o bruxismo do sono $^{1}$.

Considerando-se a etiologia multifatorial das DTM, grande parte dos estudantes não reconheceu a genética como um possível fator contribuinte: $40 \%$ do $7^{\circ}$ semestre e apenas $10 \%$ do $10^{\circ}$ semestre responderam corretamente, porém, estudos comprovam que a dor crônica é o exemplo de uma interação no ambiente genético, onde a injúria é necessária, mas existem também os fatores de suscetibilidade que podem ser herdados $^{18}$. Genes envolvidos em vias nociceptivas podem se envolver também na suscetibilidade a uma condição de dor crônica, assim como alterações genéticas e polimorfismos funcionais nesses genes podem resultar em distúrbios das vias regulatórias da dor. Estes, por sua vez, levam ao desenvolvimento de dor persistente e, portanto, contribuem para a DTM. Da mesma forma, alterações na atividade antiinflamatória podem levar as pessoas a responder diferentemente a traumas ou estresses ${ }^{19}$.

$\mathrm{Na}$ seção conhecimento, onde foram questionados sobre outros distúrbios musculoesqueléticos contribuírem para a etiologia da DTM, houve diferença estatística $(\mathrm{p}=0,016)$ quando comparadas as respostas dos dois grupos, com maior percentual de acertos para o $10^{\circ}$ semestre. Tal resultado pode ser justificado pela abordagem e estudo de fatores musculoesqueléticos e suas alterações em outras atividades clínicas, como a inter-relação das DTM com a Oclusão e a Ortodontia ${ }^{8,20}$. A DTM afeta até $15 \%$ dos adultos, com um pico de incidência e desenvolvimento dos sintomas entre 20 e 40 anos de idade, ou seja, indivíduos jovens e de meiaidade $^{5,21}$. Neste item, as alternativas ofertadas para resposta foram "indivíduos jovens", "indivíduos de meia-idade" e "indivíduos idosos". Não foram descritas no questionário as faixas etárias que cada item estaria representando, fator que poderia induzir viés ao optar por uma ou por outra alternativa. Contudo, poucos optaram por múltipla escolha, caracterizando falta de conhecimento ou má interpretação do questionamento. Os estudantes que responderam corretamente marcando as duas primeiras opções representaram apenas $20 \%$ do $7^{\circ}$ semestre e $10 \%$ do $10^{\circ}$ semestre.

Para os possíveis sintomas de DTM a maioria dos estudantes respondeu corretamente, com exceção à dor referida na região cervical, pois 90\% dos estudantes do $7^{\circ}$ semestre responderam corretamente e apenas $10 \%$ dos estudantes do $10^{\circ}$ semestre acertaram.

$\mathrm{O}$ método de diagnóstico baseado em evidências para DTM, denominado RDC/TMD, surgiu em 1992 e veio da necessidade de um sistema de diagnóstico que fosse confiável para distinguir, definir e diagnosticar diferencialmente subtipos comuns de DTM relacionadas à dor crônica, para fins de pesquisa epidemiológica e clínica $^{22}$. Em 2014, foi publicada a atualização da mudança de RDC para DC/TMD (Diagnostic Criteria for Temporomandibular Disorders) ${ }^{23,24}$. Contudo, sua validação para a língua portuguesa só ocorreu em 2019, e por este motivo os 
estudantes foram questionados ainda sobre o RDC. Apesar de tratar-se de uma ferramenta mundialmente reconhecida, uma parcela mínima dos voluntários ( $10 \%$ em cada grupo) relatou já ter conhecimento do método diagnóstico, porém, nenhum destes citou o mais importante princípio do sistema, sua abrangência biopsicossocial. A semiologia incompleta poderá subtrair dos pacientes a oportunidade de ter um adequado tratamento com consequente melhora em sua qualidade de vida ${ }^{25}$.

Para o conhecimento relacionado ao tratamento das DTM, poucos participantes demonstraram estar cientes de que identificar e remover as interferências oclusais não é eficaz, já uma porcentagem maior dos estudantes demonstrou saber que o tratamento ortodôntico não pode ser iniciado em pacientes com distúrbios na articulação. Não há estudos que fundamentem a hipótese de que a oclusão dentária interfira no desenvolvimento de DTM, por isso o tratamento baseado em técnicas irreversíveis como ajuste oclusal por desgaste seletivo, terapia ortodôntica, cirurgia ortognática ou técnicas de reabilitação oral protética no tratamento da DTM é equivocado ${ }^{20,26,27,28}$. Resultados semelhantes são observados em um estudo que avaliou o conhecimento de estudantes sobre DTM em quatro escolas de Odontologia no Brasil, onde a maioria concordou com a abordagem ortodôntica e o ajuste oclusal como ferramentas importantes para o tratamento das DTM, algo não suportado cientificamente $^{12}$.

Já sobre o tratamento invasivo da DTM, o número de estudantes do $7^{\circ}$ e $10^{\circ}$ semestre que respondeu corretamente, discordando da afirmação de que "as cirurgias de recaptura de disco são muito eficientes em casos de pacientes com deslocamento de disco com redução", foi de $70 \%$ e $40 \%$, respectivamente. Atualmente, sabese que os tratamentos conservadores devem ser os de primeira escolha, pois são eficientes para a maioria dos sintomas. Dentre eles estão as orientações de autocuidado, encaminhamentos para atendimento psicológico, fisioterapia, aplicação de laser de baixa potência, terapia medicamentosa, terapias complementares, dispositivos interoclusais rígidos e agulhamento seco ou com anestésico local para abordagens musculares $^{4,28,29}$.

Quando questionados se a toxina botulínica é um dos principais tratamentos para as DTM musculares, $90 \%$ dos estudantes do $7^{\circ}$ e $50 \%$ dos estudantes do $10^{\circ}$ semestre acertaram ao responder que discordavam do questionamento. Tal substância não apresenta papel no combate à etiologia das DTM ou do bruxismo ${ }^{30}$ e, apesar de ajudar na diminuição da dor, estudos comprovam que a toxina botulínica ainda não deve ser o tratamento de primeira escolha, pois a manipulação muscular apresenta maior eficácia na redução da sintomatologia dolorosa, além de ser uma opção mais conservadora ${ }^{31}$.

Os resultados deste estudo se assemelham também aos obtidos por outros autores que avaliaram o conhecimento de cirurgiões-dentistas poloneses $^{32}$ e indianos ${ }^{33}$. Ambos concluíram que o conhecimento de profissionais é insuficiente e que os currículos ofertados nas escolas de graduação e pós-graduação de Odontologia necessitam de revisões. Ainda, baixa carga horária de atividades práticas e teóricas acarreta menor confiança no gerenciamento das DTM ${ }^{32,33}$.

O curso de Odontologia da UFSM não apresenta oferta curricular de disciplina específica para DTM e bruxismo e os estudantes apresentaram um grau abaixo da nota média para aprovação da instituição (70\%), ficando o grupo do $7^{\circ}$ semestre com $60 \%$ e com $49 \%$ o do $10^{\circ}$. O conhecimento adquirido inicialmente mostra-se insuficiente e pode ser esquecido por não haver atividades práticas para reconhecimento, fixação e aperfeiçoamento do conteúdo abordado nas aulas teóricas. É evidente a necessidade de se dedicar 
mais atenção à DTM e ao bruxismo dentro da Odontologia, seguindo as Diretrizes Curriculares Nacionais (DCN) que valorizam a relevância social das ações de saúde e do ensino, o que implica na construção de currículos que preparem o profissional para trabalhar a partir das necessidades da população ${ }^{34,35}$. Há, ainda, a carência de políticas públicas que abordem o assunto e acolham os indivíduos que sofrem de $\mathrm{DTM}^{36}$.

A utilização de um pequeno número amostral apresenta-se como limitação deste estudo. Devido à pandemia causada pela COVID19 não foi possível a aplicação do questionário a um número maior de estudantes. Decidiu-se pela não utilização de formulários virtuais para evitar consulta a materiais didáticos. É importante salientar que os resultados deste estudo se alinham ao entendimento de que o processo ensinoaprendizado deve ser uma atividade continuada e que mais estudos devem ser estimulados, com amostras maiores e provenientes de outras instituições de ensino.

\section{CONCLUSÃO}

O estudo constatou que não há evolução do conhecimento sobre DTM e bruxismo ao longo da graduação no curso avaliado, pois o grupo de estudantes do $10^{\circ}$ semestre apresentou resultados inferiores quando comparados ao do $7^{\circ}$ semestre, que passaram recentemente pela Clínica de Oclusão, na qual os temas são abordados. Estes também apresentaram falhas no conhecimento, evidenciando a necessidade de repensar a inserção destes conteúdos durante a graduação.

\section{ABSTRACT \\ Dental students' knowledge assessment of TMD and Bruxism at UFSM}

Bruxism and temporomandibular disorders (TMD) have become increasingly frequent in clinical practice. Recognizing its mechanisms is necessary for successful management and treatment. The aim of this pilot study was to investigate the knowledge and perception about TMD and bruxism of dental undergraduate students from the Federal University of Santa Maria (UFSM). A questionnaire with 15 questions was applied to 20 students from this institution, 10 from the 7 th and 10 from the 10th semester. All evaluated students reported hearing about TMD during the Dentistry course, but $70 \%$ considered that they had little or no knowledge on the subject. Ninety percent of students reported not knowing the instrument used in research to diagnose TMD. There was no statistical difference between the evaluated groups, and the overall average of correct answers was $54 \%$. Data show little knowledge about the subjects and fragility regarding the teaching-learning process of these topics. Moreover, the non-evolution of knowledge about TMD and bruxism throughout the evaluated course is evident, as the group of students in the 10th semester showed poorer results when compared to the 7th semester, which have recently finished the discipline in which these themes are addressed.

Descriptors: Temporomandibular Joint Dysfunction Syndrome. Bruxism. Surveys and Questionnaires. Students, Dental. Educational Measurement.

\section{REFERÊNCIAS}

1. Lobbezoo F, Ahlberg J, Raphael KG, Wetselaar P, Glaros AG, Kato T, et al. International consensus on the assessment of bruxism: Report of a work in progress. J Oral Rehabil. 2018;45(11):837-44.

2. Wetselaar P, Vermaire EJH, Lobbezo F, Schuller AA. The prevalence of awake bruxism and sleep bruxism in the Dutch adult population. $\mathrm{J}$ Oral Rehabil. 2019;46(7):617-23.

3. Klasser GD. Diagnóstico e tratamento das DTM. In: De Leew R. Dor orofacial: guia de avaliação, diagnóstico e tratamento. São Paulo: Quintessence; 2013. p: 127-86.

4. Okeson JP. Tratamento das desordens 
temporomandibulares e oclusão. 6th ed. Chicago: Quintessence; 2008.

5. Gauer RL, Semidey MJ. Diagnosis and treatment of temporomandibular disorders. Am Fam Physician. 2015;91(6):378-86.

6. Borromeo GL, Trinca J. Understanding of basic concepts of orofacial pain among dental students and a cohort of general dentist. Pain Med. 2012;13(5):631-9.

7. Adibi SS, Kookal KK, Fishbeck NM, Thompson CR, Walji MF. Assessment of diagnosed temporomandibular disorders and orofacial pain conditions by predoctoral dental students: a pilot study. J Dent Educ. 2016;80(12):1450-1456.

8. Simm W, Guimarães SA. The teaching of temporomandibular disorders and orofacial pain at undergraduate level in Brazilian dental schools. J Appl Oral Sci. 2013;21(6):518-24.

9. Garcia AR, Souza V. Desordens temporomandibulares: causa de dor de cabeça e limitação da função mandibular. Rev Assoc Paul Cir Dent. 1998;52(6):480-6.

10. Costa YM, Koninck BPD, Elsaraj SM, Exposto FG, Babiloni AH, Kapos FP, et al. Orofacial pain education in dentistry: a path to improving patient care and reducing the population burden of chronic pain. J Dent Educ. 2021;85(3):349-58.

11. Siqueira SRDT. Orofacial pain and the international year. Rev Dor. 2014;15(1):1.

12. Tormes AKM, Lemos GA, Silva PLP, Forte FDS, Sousa FB, Araujo DN, et al. Temporomandibular disorders: knowledge, competency, and attitudes of predoctoral dental students. Cranio. 2020;31:1-9.

13. Universidade Federal de Santa Maria. Curso de Odontologia. Estrutura curricular. [Acesso em 7 jul. 2020]. Disponível em: http://www.ufsm.br/cursos/graduacao/santa -maria/odontologia/informacoes-do- curriculo.

14. Universidade Federal de Santa Maria. Curso de Odontologia. Disciplinas oferecidas. [Acesso em 7 jul. 2020]. Disponível em http://odontologia.ufsc.br/disciplinasoferecidas.

15. Universidade Federal de Santa Maria. Curso de Odontologia. Dados de identificação da disciplina. Programa de Ensino. [Acesso em 5 out. 2020]. Disponível em: http://deptoodt.paginas.ufsc.br/files/2012/1 1/Programa-ODT-7132-Oclus\%C3\%A3oII.pdf.

16. Blini CC, Morisso MF, Bolzan GP, Silva AMT. Relação entre bruxismo e o grau de sintomatologia de disfunção temporomandibular. Rev CEFAC. 2010;12(3):427-33.

17. Manfredini D, Peretta R, Guarda-Nardini L, Ferronato G. Predictive value of combined clinically diagnosed bruxism and occlusal features for TMJ pain. Cranio. 2010:28(2):105-13.

18. Mogil JS. Pain genetics: past, present and future. Trends Genet. 2012;28(6):258-66.

19. Smith SB, Maixner DW, Greenspan JD, Dubner R, Fillingim RB, Ohrbach R, et al. Potential genetic risk factors for chronic TMD: genetic associations from the OPPERA case control study. J Pain. 2011;12(11 Suppl):T92-101.

20. Sartoretto SC, Bello YD, Bonna AD. Evidências científicas para o diagnóstico e tratamento da DTM e a relação com a oclusão e a ortodontia. RFO UPF. 2012;17(3):352-9.

21. Liu F, Steinkeler A. Dent Clin North Am. 2013;57(3):465-79.

22. Dworkin SF, LeResche L. Research Diagnostic Criteria for Temporomandibular Disorders: review, criteria, examinations and specifications, critique. J Craniomandib 
Disord. 1992;6(4):301-55.

23. Peck CC, Goulet JP, Lobbezzo F, Schiffman EL, Alstergren $\mathrm{P}$, Anderson GC, et al. Expanding the taxonomy of the Diagnostic Criteria of Temporomandibular Disorders (DC/TMD). J Oral Rehabil. 2014;41(1):223.

24. Schiffman E, Ohrbach R, Truelove E, Look J, Anderson G, Goulet JP, et al. Diagnostic Criteria for Tempormandibular Disorders (DC/TMD) for Clinical and Research Applications: recommendations of the Internacional RDC/TMD, Consortium Network and Orofacial Pain Special Interest Group. J Oral Facial Pain Headache. 2014;28(1):6-27.

25. Klasser GD, Greene CS. Predoctoral teaching of temporomandibular disorders: a survey of US and Canadian dental schools. J Am Dent Assoc. 2007;138(2):231-7.

26. Manfredini D, Lombardo L, Siciliani G. Tempormandibular disorders and dental occlusion. A systematic review of association studies: end of an era? J Oral Rehabil. 2017;44(11):908-23.

27. Koh H, Robinson PG. Occlusal adjustment for treating and preventing temporomandibular joint disorders. J Oral Rehabil. 2004;31(4):287-92.

28. Carrara SV, Conti PCR, Barbosa JS. Statement of the 1st Consensus on Temporomandibular Disorders and Orofacial Pain. Dental Press J Orthod. 2010;15(3):114-20.

29. Sassi CF, Silva AP, Santos RKS, Andrade CRF. Tratamento para disfunções tempormandibulares: uma revisão sistemática. Audiol Commun Res. 2018;23: :e1871.

30. Patel J, Cardoso JA, Mehta S. A systematic review of botulinum toxin in the management of patients with temporomandibular disorders and bruxism. Br Dent J. 2019;226(9):667-72.

31. Guarda-Nardini L, Stecco A, Stecco C, Masiero S, Manfredini D. Myofascial pain of the jaw muscles: comparison of shortterm effectiveness of botulinum toxin injections and fascial manipulation technique. Cranio 2012;30(2):95-102.

32. Osiewicz M, Kojat P, Gut M, Kazibudzka Z, Pytko-Polonczyk J. Self-perceived dentists' knowledge of temporomandibular disorders in Krakow: a pilot study. Pain Res Manag. 2020(6):1-6.

33. Patil S, Iyenger AR, Ramneek. Assessment of knowledge, attitude and practices of dental practitioners regarding temporomandibular joint disorders in India. JCRI. 2016;3(2):64-71.

34. Morita MC, Haddad AE. Interfaces da área da Educação e da Saúde na perspectiva da formação e do trabalho das equipes de Saúde da Família. In: Moysés ST, Kriger L, Moysés SJ (Coord). Saúde bucal das famílias: trabalhando com evidências. São Paulo: Artes Médicas; 2008. p.268-76.

35. Zilbovicius C, Araujo ME, Botazzo C, Frias AC, Junqueira SR, Junqueira CR. A paradigm shift in predoctoral dental curricular in brazil: evaluating the process of change. J Dent Educ. 2011;75(4):557-64.

36. Morita MC, Kriger L. Mudanças nos cursos de Odontologia e a interação com o SUS. Rev ABENO. 2004;4(1):17-21.

\section{Correspondência para:}

Tatiana Bernardon Silva

e-mail: tabernardon@hotmail.com

Av. Roraima, 1000 - Prédio 26F

Cidade Universitária - Bairro Camobi

97105-900 Santa Maria/RS 